\title{
¿EXIGE EL DELITO DE FUGA, PRESCRITO Y SANCIONADO EN EL ARTÍCULO 195 DE LA LEY DE TRÁNSITO, QUE EL CONDUCTOR SE HAYA ENCONTRADO EN ESTADO DE EBRIEDAD? COMENTARIO A LA SENTENCIA ROL 3582-2015, DE LA CORTE DE APELACIONES DE SANTIAGO*
}

\author{
JAVIER ESCOBAR VEAS**
}

\section{INTRODUCCIÓN}

El presente comentario versa sobre la sentencia rol 3582-2015, de la Corte de Apelaciones de Santiago, de fecha 31 de diciembre de 2015.

En este caso, el Ministerio Público presentó acusación en contra del imputado por el delito contenido en el artículo 195 de la Ley $\mathrm{N}^{\circ}$ $18.290^{11-22}$, que establece la Ley de Tránsito, cuyo texto refundido, coordinado y sistematizado está contenido en el Decreto con Fuerza de Ley $\mathrm{N}^{\circ} 1 / 2009$, del Ministerio de Transporte y Telecomunicaciones.

El $2^{\circ}$ Tribunal de Juicio Oral en lo Penal de Santiago, en sentencia RIT 482-2015, absolvió al imputado, estimando que los hechos contenidos en la acusación no podían subsumirse en el tipo penal invocado. El tribunal sostuvo como motivo absolutorio principal que el delito de fuga desde el lugar del accidente, prescrito y sancionado en el artículo 195, exige para su configuración que el conductor imputado se haya encontrado en estado de ebriedad.

En contra de la sentencia absolutoria, el Ministerio Público presentó un recurso de nulidad, elevándose los antecedentes ante la Corte de Apelaciones de Santiago. La Corte rechazó el libelo interpuesto, haciendo suyo el motivo absolutorio precedentemente indicado ${ }^{3}$.

Fecha de recepción: 4 de agosto de 2017.

Fecha de aceptación: 13 de marzo de 2018.

** Doctorando en Derecho, Università Luigi Bocconi; Magíster en Derecho Penal y Licenciado en Ciencias Jurídicas y Sociales, Universidad Diego Portales; Profesor de Derecho Penal, Universidad Mayor (Chile). Correo electrónico: javier.escobar@mail.udp.cl

1 Toda referencia normativa, sin indicar cuerpo legal, debe entenderse realizada a la Ley $\mathrm{N}^{\circ} 18.290$. A pesar de que la conducta incriminada no consiste únicamente en escapar desde el lugar del accidente, para efectos de simplificar su denominación nos referiremos a este ilícito como delito de fuga.

3 Es pertinente señalar que la sentencia de instancia, además, contiene un motivo absolutorio subsidiario. El tribunal sostuvo que, en caso de que el razonamiento absolutorio principal fuese desestimado, de todas maneras el acusado debiese ser absuelto, pues el delito contemplado en el artículo 195 exige, como requisito para su configuración, que el imputado haya sido "responsable" del accidente en cuestión, elemento que no concurría en el caso de autos. Considerando que la sentencia de la Corte de Apelaciones no se pronunció sobre aquel motivo absolutorio, el mismo no será abordado en esta oportunidad. 


\section{1) Hechos acreditados y razonamiento de la Corte de APELACIONES DE SANTIAGO}

Con fecha 10 de noviembre de 2015 se realizó el juicio oral en contra del imputado, por el delito contemplado en el artículo 195.

Los hechos materia de la acusación fueron: "El día 1 de diciembre de 2014, aproximadamente a las 01:10 horas, en circunstancias que el imputado D. A. C. S. conducía el vehículo taxi básico (...) por Avenida Santa María al llegar a Avenida La Paz, de la comuna de Recoleta, colisionó y atropelló a la víctima, J. C. C. R., quien sorpresivamente atravesó dicha avenida, en estado de ebriedad, sin respetar el normal flujo vehicular, que lo hacía con luz verde de semáforo. Producto de la colisión J. C. C. R. quedó tirada y lesionada en el lugar, procediendo el acusado (...) a darse a la fuga del lugar, sin prestarle ayuda alguna, ni dar cuenta de lo ocurrido a la autoridad policial más cercana. Producto del atropello dońa J. C. C. R. falleció por politraumatismo".

Tras haber valorado las pruebas rendidas por los intervinientes, el tribunal tuvo por acreditados los hechos descritos en la acusación fiscal, señalando que no había sido controvertido el hecho que el día 1 de diciembre de 2014, el acusado conducía un automóvil tipo taxi por Avenida Santa María y que, al llegar a la intersección con Avenida La Paz, atropelló a una mujer que cruzó la calzada por una vía no habilitada, siendo la imprudencia de esta lo que causó el atropello y su consiguiente fallecimiento. Sin perjuicio de lo anterior, el tribunal señala que lo central en el caso de autos es la posibilidad de subsumir o no los hechos precedentemente descritos en el tipo penal por el cual se había deducido acusación, operación que, en su opinión, no podía efectuarse, toda vez que el ilícito del artículo 195 exige, como elemento típico, que el conductor imputado se haya encontrado en estado de ebriedad.

En contra de la sentencia absolutoria el Ministerio Público dedujo un recurso de nulidad, basado en la causal contemplada en el artículo 373 letra b) del Código Procesal Penal, esta es, por errónea aplicación del derecho.

La Corte de Apelaciones de Santiago, conociendo del recurso interpuesto, resolvió rechazarlo, estimando efectivamente que los hechos acreditados no podían subsumirse en el delito de fuga del artículo 195 por la razón esgrimida por el tribunal.

El razonamiento desarrollado por la Corte de Apelaciones fue el siguiente:

a) Que a objeto de dilucidar la cuestión planteada, vale decir, si es o no posible realizar el ejercicio de subsunción requerido, es pertinente recordar que la Ley $\mathrm{N}^{\circ} 20.770$ tuvo como origen un mensaje presentado por el Ejecutivo. Este mensaje dio comienzo a la tramitación del proyecto de ley denominado "Modifica la Ley del Tránsito, en lo 
que se refiere al delito de Manejo en Estado de Ebriedad, causando Lesiones Graves, Gravísimas o muerte”, el cual proponía incorporar diversas modificaciones relativas a las sanciones aplicables a este tipo de delitos.

b) El mensaje señalaba que la baja extensión de la pena a aplicar, sumada a la existencia de penas sustitutivas, tenía como consecuencia que los autores de este delito cumplieran sus penas en libertad, lo cual había generado en la población una sensación de impunidad respecto de este tipo de ilícitos. Para efectos de combatir este problema, se proponían las siguientes modificaciones:

1. Aumentar las penas para el delito de conducción en estado de ebriedad causando lesiones graves, gravísimas o muertes.

2. Crear una hipótesis calificada del delito, la cual se aplicaría en aquellos casos en que el responsable huyera del lugar del accidente, fuera reincidente o se tratase de un conductor profesional en el ejercicio de sus funciones.

3. Introducir reglas especiales para la determinación de la pena.

4. Introducir reglas especiales para la aplicación de la Ley $\mathrm{N}^{\circ}$ $18.216^{4}$.

c) Que resulta pertinente tener en cuenta que, conforme al artículo 201 $\mathrm{N}^{\circ} 15$, son infracciones o contravenciones menos graves, entre otras, no cumplir las obligaciones que impone el artículo 176, mismos deberes cuya omisión sanciona como delito el artículo $195^{5}$.

d) "Que, así las cosas, la interpretación sistemática, histórica y teleológica de la normativa precedentemente aludida determina necesariamente concluir que efectivamente y tal como razonaron los sentenciadores del fondo, el ámbito de aplicación del artículo 195 de la Ley 18.290 se encuentra íntimamente asociado a la conducción de un vehículo motorizado bajo los efectos del alcohol o en estado de ebriedad, puesto que solo tal criterio exegético dilucida la existencia de preceptos que en forma paralela asignan distinta gravedad a idénticas conductas omisivas y la razonabilidad y justificación de la gran entidad de la pena asignada al comportamiento objetivo de desatención de los deberes de detención de la marcha, de prestación de socorro y de denuncia a la autoridad policial que el aludido precepto legal contiene" 6 .

Corte de Apelaciones de Santiago. 31 de diciembre de 2015. Rol No 3.582-2.015. Disponible en: https://oficinajudicialvirtual.pjud.cl/frameInv.php [fecha de visita: 1 de agosto de 2017]. Considerando Tercero.

Corte de Apelaciones de Santiago. Rol No 3.582-2.015. Considerando Cuarto.

Corte de Apelaciones de Santiago. Rol No 3.582-2.015. Considerando Quinto. 


\section{2) COMENTARIO}

Con fecha 16 de septiembre de 2014 fue publicada en el Diario Oficial la Ley $\mathrm{N}^{\circ}$ 20.770, conocida mediáticamente como "Ley Emilia", cuerpo normativo que, entre otras modificaciones, sustituyó íntegramente el texto del artículo 195, estableciendo además reglas especiales sobre determinación y sustitución de penas ${ }^{7}$. La intención originaria de este nuevo cuerpo normativo, tal como se indicó en el mensaje del Ejecutivo, fue sancionar con mayor severidad el delito de manejo en estado de ebriedad con resultado de muerte o de lesiones gravísimas (artículo $397 \mathrm{~N}^{\circ} 1$ del Código Penal).

Actualmente, el artículo 195 dispone:

"Artículo 195.- El incumplimiento de la obligación de dar cuenta a la autoridad de todo accidente en que solo se produzcan daños, señalada en el artículo $168^{8}$, será sancionado con multa de tres a siete unidades tributarias mensuales y con la suspensión de la licencia hasta por un mes.

El incumplimiento de la obligación de detener la marcha, prestar la ayuda posible y dar cuenta a la autoridad de todo accidente en que se produzcan lesiones, señalada en el artículo $176^{9}$, se sancionará con la pena de presidio menor en su grado medio, inhabilidad perpetua para conducir vehículos de tracción mecánica y multa de siete a diez unidades tributarias mensuales.

$\mathrm{Si}$ en el caso previsto en el inciso anterior las lesiones producidas fuesen de las señaladas en el número $1^{\circ}$ del artículo 397 del Código Penal o se produjese la muerte de alguna persona, el responsable será castigado con la pena de presidio menor en su grado máximo, inhabilidad perpetua para conducir vehículos de tracción mecánica, multa de once a veinte unidades tributarias mensuales y con el comiso del vehículo con que se ha cometido el delito, sin perjuicio de los derechos del tercero propietario,

7 Matus, Jean Pierre; Ramírez, María Cecilia (2017) Manual de Derecho Penal Chileno, Parte Especial. Valencia: Editorial Tirant lo Blanch, p. 220.

8 "Artículo 168.- En todo accidente del tránsito en que se produzcan dańos el o los participantes estarán obligados a dar cuenta de inmediato a la autoridad policial más próxima.

Se presumirá la culpabilidad del o de los que no lo hicieren y abandonaren el lugar del accidente.

Asimismo, se presumirá la responsabilidad del conductor que no cumpla lo establecido en el artículo 176 y abandonare el lugar del accidente.

En todo caso, para hacer efectivos los seguros de daños a terceros o propios, el interesado deberá informar el siniestro mediante declaración jurada simple presentada ante la respectiva compañía aseguradora, y no se requerirá de otros actos o documentos expedidos por la autoridad policial, tales como constancias o denuncia".

9 "Artículo 176.- En todo accidente del tránsito en que se produzcan lesiones o muerte, el conductor que participe en los hechos estará obligado a detener su marcha, prestar la ayuda que fuese posible y dar cuenta a la autoridad policial más inmediata, entendiéndose por tal cualquier funcionario de Carabineros que estuviere próximo al lugar del hecho, para los efectos de la denuncia ante el Tribunal correspondiente". 
que podrá hacer valer conforme a las reglas generales del Código Procesal Penal. Para los efectos de determinar la pena prevista en este inciso, será aplicable lo dispuesto en los artículos 196 bis y 196 ter de esta ley.

Las penas previstas en este artículo se impondrán al conductor conjuntamente con las que le correspondan por la responsabilidad que le pueda caber en el respectivo delito o cuasidelito, de conformidad con lo dispuesto en el artículo 74 del Código Penal”.

En cuanto a la estructura del tipo penal, esta puede resumirse de la siguiente manera:

a. Accidentes donde solo se han ocasionado daños: Conforme al inciso primero, el conductor involucrado deberá dar aviso inmediato a la autoridad policial más próxima. La omisión de tal obligación será sancionada con la pena de multa de tres a siete unidades tributarias mensuales y la suspensión de la licencia de conducir hasta por un mes.

b. Accidentes donde se han ocasionado lesiones o muertes: De acuerdo a los incisos segundo y tercero, el conductor deberá detener su marcha, prestar la ayuda que fuese posible y dar cuenta a la autoridad policial más inmediata. La omisión de tal obligación, conforme establecen los mismos incisos, dependerá de los específicos resultados lesivos ocasionados.

De esta forma, el delito se erige como una hipótesis de omisión propia, toda vez que es el propio tipo penal el que expresamente describe la omisión punible, respecto de la cual no existe una contrafigura en forma de delito comisivo ${ }^{10}$.

En relación al caso que se comenta, la discusión jurídica planteada versó sobre aquellos elementos o condiciones que debe reunir el sujeto activo del delito de fuga. La Corte de Apelaciones, tras un complejo proceso interpretativo, arribó a la conclusión de que, para efectos de apreciar un sujeto activo idóneo en el delito indicado, es necesario que el conductor imputado se haya encontrado en estado de ebriedad.

Ahora, en cuanto al contenido de la sentencia, destaca en primer lugar la metodología interpretativa utilizada. Al respecto, cabe recordar que el proceso de "interpretación legal consiste en atribuir un significado a la disposición que se interpreta”. Por tanto, los criterios interpretativos son la respuesta a dónde buscar el significado de la disposición en estudio ${ }^{11}$. Sobre la interpretación de las normas penales, la doctrina reconoce que la sujeción del juez a la ley, en virtud del principio de legalidad, es más

10 Cury, Enrique (2011) Derecho Penal, Parte General, 10a edición. Santiago: Editorial Universidad Católica, p. 679.

11 Rodríguez-Toubes, Joaquín (2013). "El criterio histórico en la interpretación jurídica”. Dereito: Revista Xuridica da Universidade de Santiago de Compostela, V. 22, No Ext, pp. 599-632, p. 605. Disponible en: http://www.usc.es/revistas/index.php/dereito/article/ view/1184/1545 [fecha de visita: el 13/12/2017]. 
acentuada que en otros campos del ordenamiento jurídico ${ }^{12}$. No obstante, igualmente son aplicables "las reglas o criterios empleados en los demás campos jurídicos (...), siempre y cuando no implique su uso una violación del principio de la legalidad"13. En este sentido, se ha sostenido que la aproximación literal o formalista constituye el primer paso interpretativo ${ }^{14}$, tal como dispone el artículo 19, inciso primero, del Código Civil ${ }^{15}$. Si el intérprete estima que el sentido de la ley es claro, el proceso interpretativo finalizará allí. En cambio, si el examen literal resulta infructuoso, debido a que no es posible aclarar el sentido correcto de la disposición normativa, o el sentido obtenido genera resultados abiertamente absurdos o indeseables, el intérprete podrá recurrir a otras fuentes o herramientas, entre las cuales se encuentra el elemento histórico y teleológico ${ }^{16}$.

La metodología utilizada por la Corte, en el caso concreto, corresponde a una "histórica-teleológica". En efecto, cabe suponer que, en su opinión, la interpretación literal del artículo 195, y su consecuente aplicación al caso concreto, se apreció como injusta y desproporcionada, especialmente considerando que la conducta del imputado ni siquiera había generado un riesgo prohibido, sino que, todo lo contrario, había cumplido con todas las normas de cuidado establecidas. Ante este escenario, la Corte, efectuando un esfuerzo interpretativo, decidió recurrir a antecedentes históricos (legislativos ${ }^{17}$ ) con el objetivo de aprehender la ratio legis de la norma en estudio, concluyendo, como ya se ha señalado, que el sujeto activo del delito de fuga debe haberse encontrado en estado de ebriedad.

Ahora, ¿es posible considerar, en términos interpretativos, que la tesis planteada en la sentencia sea correcta?

12 Hurtado, José (1974) "La interpretación de la ley en el Derecho Penal". Revista de Derecho PUCP, $\mathrm{N}^{\circ} 32$, pp. 162-177, p. 176. Disponible en: http://revistas.pucp.edu.pe/index.php/ derechopucp/article/view/6145 [fecha de visita: el 13/12/2017].

13 Hurtado (1974) 167. Sobre el principio de legalidad en Derecho Penal, en extenso Roxin, Claus (1997) Derecho Penal, Parte General. Traducido por Luzón, Diego; Díaz y García, Manuel; de Vicente, Javier. Madrid: Editorial Civitas. Vol. I, pp. 134 y ss.

14 Hurtado (1974) 167; Camaño, Antonio (1951) "Interpretación de las leyes penales". Anuario de Derecho Penal y Ciencias Penales, Fascículo 2, pp. 243-252, p. 247. Disponible en: https://www.boe.es/publicaciones/anuarios_derecho/anuario.php?id=P_1951_ANUARIO_DE_DERECHO_PENAL_Y_CIENCIAS_PENALES\&fasc=2 [fecha de visita: el $13 / 12 / 2017]$.

15 "Art. 19. Cuando el sentido de la ley es claro, no se desatenderá su tenor literal, a pretexto de consultar su espíritu.

16 Camaño (1951) 247. Tal idea pareciera subyacer al inciso segundo del artículo 19 del Código Civil, el cual dispone: "Pero bien se puede, para interpretar una expresión obscura de la ley, recurrir a su intención o espíritu, claramente manifestados en ella misma, o en la historia fidedigna de su establecimiento".

17 Sobre la utilización de materiales prelegislativos y otros antecedentes de similar índole, ver Rodríguez-Toubes (2013) 613 y ss. 
Según nuestra opinión, la respuesta es negativa. Lo anterior debido a que la historia fidedigna de la Ley $\mathrm{N}^{\circ} 20.770$, principal antecedente invocado por la Corte, en realidad no respalda ni justifica las conclusiones contenidas en la sentencia. El error interpretativo se genera en el momento en que no se considera la historia legislativa en su totalidad y, en cambio, se atribuye decisiva importancia a documentos no representativos de la misma, según se explicará a continuación.

Como primer elemento, resulta imperioso destacar que el delito de fuga fue incorporado a nuestro ordenamiento jurídico por la Ley $\mathrm{N}^{\circ}$ 20.068, publicada en diciembre de 2005, y no por la Ley $\mathrm{N}^{\circ} 20.770$. Aquel cuerpo normativo introdujo un nuevo artículo 196 D 1 a la Ley de Tránsito, del siguiente tenor:

"El incumplimiento, a sabiendas, de lo señalado en el artículo 173 será sancionado con multa de 3 a 7 unidades tributarias mensuales y con la suspensión de su licencia hasta por un mes. El incumplimiento, a sabiendas, de lo señalado en el artículo 183 será sancionado con la suspensión de la licencia de conducir por un plazo máximo de 12 meses y si el juez así lo estimare, presidio menor en grado mínimo a medio, salvo que las lesiones producidas tengan el carácter de leves, en cuyo caso se aplicará la sanción del inciso primero del artículo 196 C”.

La tramitación legislativa de esta nueva norma no estuvo exenta de complicaciones. Durante el Primer Trámite Constitucional la Cámara de Diputados propuso incorporar un nuevo inciso segundo al artículo 183, el cual señalaba:

"El incumplimiento de lo dispuesto en este artículo podrá ser sancionado con la cancelación de la licencia de conducir y con la pena de presidio menor en sus grados medio a máximo, salvo que las lesiones producidas tengan el carácter de leves".

No obstante, durante el Segundo Trámite Constitucional el Senado modificó la propuesta de la Cámara de Diputados, pues consideró asistemática la incorporación de este nuevo tipo penal fuera del Título XVII, apartado que establece los delitos y cuasidelitos de la Ley de Tránsito. Además, el Senado rebajó la sanción propuesta por la Cámara de Diputado, argumentando que aquella era desproporcionada pues la nueva figura tendría la misma pena corporal que el homicidio culposo ${ }^{18}$. La pena privativa de libertad fue disminuida a presidio menor en sus grados mínimo a medio, facultándose al juez para no imponerla en el caso concreto.

Cuando se refundió y sistematizó el texto de la Ley de Tránsito, el artículo 196 D 1 pasó a ser el 195.

De esta forma, resulta incuestionable que el delito de fuga, contenido originalmente en el artículo 196 D 1, y luego en el 195, no fue creado

18 Historia de la Ley No 20.068, Biblioteca del Congreso Nacional, pp. 223 y 234. 
por la Ley $\mathrm{N}^{\circ}$ 20.770. Por el contrario, esta solo se limitó a sustituir el texto de esta última norma por uno similar, aumentando las penas contempladas.

Por tanto, antes de la entrada en vigencia de la Ley $\mathrm{N}^{\circ} 20.770$, el conductor que abandonaba el lugar del accidente, habiendo provocado lesiones menos graves o superiores, era sancionado con suspensión de su licencia de conducir hasta por un plazo de 12 meses y, si el juez así lo estimaba, con la pena de presidio menor en sus grados mínimo a medio ${ }^{19} 20$.

Ahora, en lo que respecta al mensaje que dio origen al proyecto de ley que concluyó en la publicación de la Ley $\mathrm{N}^{\circ} 20.770$, efectivamente este tenía como finalidad elevar las sanciones de los delitos de manejo en estado de ebriedad con resultado de lesiones gravísimas o muerte. La intención del proyecto era hacerse cargo de la sensación de impunidad que se había generado en la sociedad ante el delito de manejo en estado de ebriedad con resultado de muerte, ya que la baja extensión y la concesión de penas sustitutivas llevaba finalmente a que sus autores cumplieran las penas en libertad. En opinión del Ejecutivo, "la sociedad no comprende cómo una persona que voluntariamente bebió hasta embriagarse, que voluntariamente condujo un vehículo y lesionó o incluso mató a una persona, no sea considerado autor de un delito grave que le impida obtener su libertad"21.

Esta "sensación de impunidad" ha sido explicada por Matus en los siguientes términos: "Pareciera que la ley ofreciese para el responsable de desgracias como las sufridas por la pequeña Emilia un tratamiento penal que consideraría adecuadamente la gravedad del peligro puesto por la condición en estado de ebriedad (...). El problema es que, en concreto, la aplicación a los hechos de las disposiciones de la Ley de Tránsito, del Código Penal, en relación con las circunstancias atenuantes concurrentes, y de la Ley $\mathrm{N}^{\circ} 18.216$, sobre penas sustitutivas, tiende a producir efectos prácticos que desdibujan las diferenciaciones que, en abstracto, pretende hacer la legislación. Y el caso de la menor Emilia Silva sirve también para ilustrar esa pérdida de diferenciación en las sanciones: tras un juicio oral, el responsable del hecho fue condenado exclusivamente a una pena de dos años de presidio menor en su grado medio, (...) pena a su vez sustituida por su remisión condicional (...). Así, además de perderse la diferenciación entre los cuasidelitos comunes y la conducción en estado de ebriedad, las penas impuestas en este caso $-\mathrm{y}$ en muchos otros similares,

\footnotetext{
19 Matus, Jean Pierre (2014) "Ley Emilia”. Revista Doctrina y Jurisprudencia Penal, Año 5, edición especial, pp. 101-113, p. 102.

20 Sobre el delito del antiguo artículo 195, ver van Weezel, Alex (2014) "Solidaridad en el tráfico motorizado. El delito de omisión de auxilio en caso de accidente". Revista Doctrina y Jurisprudencia Penal, Año 5, edición especial, pp. 191-204.

$21 \quad$ Historia de la Ley $N^{\circ}$ 20.770, Biblioteca del Congreso Nacional, p. 4.
} 
como el de un conocido futbolista- tienden, en los hechos, a eliminar el carácter penal de las sanciones, asemejándolas demasiado a las impuestas por los Juzgados de Policía Local respecto de la reiteración de infracciones de tránsito graves" 22 .

Entre otras modificaciones, el proyecto original proponía incorporar un nuevo inciso cuarto al artículo 196, estableciendo tres circunstancias agravantes, las cuales tendrían como efecto que, para responsable del delito de manejo en estado de ebriedad con resultado de muerte o lesiones gravísimas, la pena a imponer sería de presidio mayor en su grado mínimo. Una de tales circunstancias agravantes era darse a la fuga del lugar del accidente sin prestar auxilio a la víctima ${ }^{23}$. En efecto, el artículo $1^{\circ} \mathrm{N}^{\circ} 2$ del proyecto de ley original disponía:

"2) Incorpórase el siguiente inciso cuarto, nuevo, al artículo 196:

$\mathrm{Al}$ autor del delito previsto en el inciso precedente se le impondrá la pena de presidio mayor en su grado mínimo y multa de ocho a veinte unidades tributarias mensuales, además de la pena de inhabilidad perpetua para conducir vehículos de tracción mecánica si concurrieren alguna de las circunstancias siguientes:

$1^{\circ}$ Si el responsable huyere del lugar del accidente y no prestare ayuda a la víctima;

$2^{\circ}$ Si el responsable hubiese sido condenado anteriormente por alguno de los delitos previstos en este artículo;

$3^{\circ}$ Si el delito hubiere sido cometido por un conductor cuya profesión u oficio consista en el transporte de personas o bienes y hubiere actuado en el ejercicio de sus funciones" 24 .

Es imprescindible destacar que el proyecto de ley original no derogaba el artículo 195, de modo tal que la figura típica contemplada en esta norma seguiría vigente para todos los efectos legales. Debido a lo anterior, el sistema propuesto por el mensaje era el siguiente:

a. Si el responsable de un delito de manejo en estado de ebriedad con resultado de muerte o lesiones gravísimas se daba la fuga, esta configuraría la nueva agravante propuesta, elevándose la pena corporal a presidio mayor en su grado mínimo.

\footnotetext{
22 Matus (2014) 102.

23 Matus señala que el proyecto original, para lograr sus objetivos, proponía, entre otras, la siguiente modificación legal: "b) Establecer un delito calificado penado con presidio mayor en su grado mínimo (5 años y 1 día a 10) cuando, junto con el manejo en estado de ebriedad causando muerte, el autor se diese a la fuga, fuese reincidente o conductor con licencia profesional". Matus (2014) 109.

24 Historia de la Ley $\mathrm{N}^{\circ} 20.770$, Biblioteca del Congreso Nacional, p. 5.
} 
b. En cambio, si el conductor que se daba a la fuga no se encontraba en estado de ebriedad, lo que correspondería sería discutir la eventual configuración del delito autónomo del artículo 195.

El sistema propuesto por el mensaje fue modificado durante la tramitación legislativa. Específicamente, la Comisión de Constitución, Legislación, Justicia y Reglamento del Senado decidió suprimir la fuga del lugar del accidente del listado de agravantes del inciso cuarto del artículo 196. El Ejecutivo propuso, en su lugar, derogar el artículo 195 e incorporar cuatro nuevos incisos al artículo 176, los cuales regularían el delito de fuga $^{25}$. Tal propuesta, sin embargo, fue advertida como asistemática, toda vez que el nuevo tipo penal se encontraría fuera del Título XVII, apartado que establece los delitos del ramo. Fue entonces cuando se propuso sustituir el texto del artículo 195, incorporando a este el contenido de los nuevos incisos propuestos por el Ejecutivo, idea que fue finalmente acogida.

Tras haber analizado la historia y evolución legislativa del delito de fuga, es posible arribar a las siguientes conclusiones:

a. El proyecto de ley original no sustituía ni derogaba el artículo 195, sino que proponía incorporar la fuga como una agravante aplicable al delito de manejo en estado de ebriedad causando lesiones gravísimas o muerte. Lo anterior significa que, en el sistema propuesto por el mensaje, la fuga podía configurar el ilícito autónomo del artículo 195 o una agravante en el tipo penal de manejo en estado de ebriedad.

b. De esta forma, queda demostrado que el delito de fuga en ningún momento se concibió como una figura subordinada al manejo en estado de ebriedad causando lesiones gravísimas o muerte.

c. La idea de sustituir el artículo 195 surgió únicamente como una respuesta a una indicación presentada por el Ejecutivo, la cual fue denunciada como asistemática, pues generaba problemas de coherencia interna al proponer incorporar un tipo penal fuera del Título correspondiente.

25 El texto de estos nuevos incisos era el siguiente:

"El incumplimiento de lo seńalado en el inciso anterior, se sancionará con la pena de presidio menor en su grado máximo y con el comiso del vehículo, sin perjuicio de los derechos del tercero propietario, que podrá hacer valer conforme a las reglas generales del Código Procesal Penal.

La pena prevista en el inciso anterior se impondrá al conductor conjuntamente con la que le corresponda por la responsabilidad que le pueda caber en el respectivo delito o cuasidelito, de conformidad con lo dispuesto en el artículo 74 del Código Penal.

Para los efectos de determinar la pena en este artículo, será aplicable lo dispuesto en el artículo 196 bis y 196 ter de esta ley.

No se aplicarán las reglas especiales de determinación de la pena, señaladas en el inciso anterior si, como resultado del accidente, solo se ocasionaren lesiones menos graves, leves o daño". 
Como último argumento, cabe resaltar que adscribir a la tesis planteada en la sentencia tendría consecuencias más allá del artículo 195, obligándonos a interpretar todas las modificaciones efectuadas por la Ley $\mathrm{N}^{\circ} 20.770$ en la misma clave hermenéutica. A modo de ejemplo, esto implicaría que el delito del artículo 195 bis también sería un ilícito subordinado al manejo en estado de ebriedad. Lo anterior significaría que únicamente un conductor que se hubiere encontrado en dicho estado podría cometer este delito, vale decir, un tipo penal cuya conducta consiste en negarse a la realización de exámenes destinados a verificar la existencia de sustancias prohibidas, solamente podría ser cometido por un conductor que las haya consumido previamente, interpretación contra la cual la doctrina se ha pronunciado expresamente ${ }^{26}$.

\section{CONCLUSIONES}

a. El esfuerzo interpretativo realizado por la Corte de Apelaciones puede explicarse en atención a que, en el caso concreto, la aplicación del artículo 195, y la imposición de las sanciones en él contenidas, se consideró injusta y desproporcionada, sobre todo en atención a que el imputado había cumplido todas y cada una de las normas de cuidado establecidas.

b. Sin embargo, la tesis comentada, que concibe al delito de fuga como un ilícito subordinado al manejo en estado de ebriedad, no puede ser compartida.

c. La Corte de Apelaciones basa su tesis en el estudio de la historia legislativa de la Ley $\mathrm{N}^{\circ} 20.770$. No obstante, esta no respalda las premisas expuestas en la sentencia. En efecto, la fuga desde el lugar del accidente, en el sistema propuesto por el proyecto de ley original, podía configurar el delito autónomo del artículo 195 o una agravante (en el delito de manejo en estado de ebriedad con resultado de lesiones gravísimas o muertes). Lo anterior demuestra que, para efectos del artículo 195, la condición etílica del conductor era irrelevante.

d. Sin perjuicio de lo anterior, la no-aplicación del delito de fuga a este caso concreto podría haberse fundamentado en base a un estudio más acabado sobre aquellos elementos o condiciones que permiten configurar un sujeto activo idóneo en este ilícito, reflexión que, por supuesto, excede los márgenes del presente comentario.

26 Falcone, Diego (2015) "El delito de negativa injustificada de un conductor a someterse a los exámenes de detección de alcohol o sustancias estupefacientes o psicotrópicas". Revista de Derecho de la Pontifica Universidad Católica de Valparaíso, V. 46, N 1, pp. 143-169, pp. 157 y 158. Disponible en: http://www.scielo.cl/pdf/rdpucv/n44/a05.pdf [fecha de visita: el 2/6/2017]. 


\section{BibLiOgRAFÍA}

Historia de la Ley $\mathrm{N}^{\circ}$ 20.770, Biblioteca del Congreso Nacional. Disponible en: http://www.bcn.cl/historiadelaley/nc/historia-de-laley/4318/

Camaño, Antonio (1951) "Interpretación de las leyes penales". Anuario de Derecho Penal y Ciencias Penales, Fascículo 2, pp. 243-252. Disponible en: https://www.boe.es/publicaciones/anuarios_derecho/ anuario.php?id=P_1951_ANUARIO_DE_DERECHO_PENAL_Y_ CIENCIAS_PENALES $\&$ fasc $=2$

Cury, Enrique (2011) Derecho Penal, Parte General, 10a edición. Santiago: Editorial Universidad Católica.

Falcone, Diego (2015) "El delito de negativa injustificada de un conductor a someterse a los exámenes de detección de alcohol o sustancias estupefacientes o psicotrópicas". Revista de Derecho de la Pontifica Universidad Católica de Valparaíso, V. 46, No 1, pp. 143169. Disponible en: http://www.scielo.cl/pdf/rdpucv/n44/a05.pdf

Hurtado, José (1974) "La interpretación de la ley en el Derecho Penal". Revista de Derecho PUCP, $\mathrm{N}^{\circ} 32$, pp. 162-177. Disponible en: http://revistas.pucp.edu.pe/index.php/derechopucp/article/ view/6145

Matus, Jean Pierre; Ramírez, María Cecilia (2017) Manual de Derecho Penal Chileno, Parte Especial. Valencia: Editorial Tirant lo Blanch.

Matus, Jean Pierre (2014) "Ley Emilia". Revista Doctrina y Jurisprudencia Penal, Año 5, edición especial, pp. 101-113.

Rodríguez-Toubes, Joaquín (2013) "El criterio histórico en la interpretación jurídica”. Dereito: Revista Xurídica da Universidade de Santiago de Compostela, V. 22, No Ext, pp. 599-632. Disponible en: http://www.usc.es/revistas/index.php/dereito/article/view/1184/1545

Roxin, Claus (1997) Derecho Penal, Parte General. Traducido por Luzón, Diego; Díaz y García, Manuel; de Vicente, Javier. Madrid: Editorial Civitas. Vol. I.

VAn WeEzel, Alex (2014) "Solidaridad en el tráfico motorizado. El delito de omisión de auxilio en caso de accidente". Revista Doctrina y Jurisprudencia Penal, Año 5, edición especial, pp. 191-204.

\section{JURISPRUDENCIA CITADA}

Corte de Apelaciones de Santiago. 31 de diciembre de 2015. Rol N ${ }^{\circ}$ 3.582-2.015. Disponible en: https://oficinajudicialvirtual.pjud.cl/ frameInv.php [fecha de visita: 1 de agosto de 2017]. 